INPLASY

PROTOCOL

To cite: Liu et al. Effects of Physical Exercise on Shoulder Motion and Upper Limb

Function after Surgery of

Breast Cancer Patients: A

Meta-analysis. Inplasy protocol 202160018. doi:

10.37766/inplasy2021.6.0018

Received: 06 June 2021

Published: 06 June 2021

Corresponding author:

Cong Liu

1921111043@sus.edu.cn

Author Affiliation:

Shanghai University of Sport.

Support: 11DZ2261100.

Review Stage at time of this submission: Data analysis.

Conflicts of interest:

None declared.

\section{Effects of Physical Exercise on Shoulder Motion and Upper Limb Function after Surgery of Breast Cancer Patients: A Meta-analysis}

Liu, C1; Chen, R2; Zheng, $\mathrm{XH}^{3}$; Wang, $\mathrm{X}^{4}$.

Review question / Objective: To systematically evaluate the intervention effect of physical exercise on shoulder range of motion and upper limb function in patients with breast cancer after surgery.

Condition being studied: Breast cancer (BC) is the most common disease in women and the leading cause of cancer death in women. With the progress of medical treatment, the survival rate of breast cancer patients after 5 years has been as high as $\mathbf{9 0} \%$, but the complications caused by surgery and chemotherapy are more serious.Studies have shown that up to $67 \%$ of breast cancer patients experience reduced shoulder mobility and upper limb dysfunction following treatment. Long-term dysfunction can lead to chronic pain, decreased muscle strength, lymphedema, cardiopulmonary dysfunction, and even permanent disability.However, sports can improve shoulder joint activity and upper limb function in patients with breast cancer, therefore, proposed using Meta analysis method, the exercise therapy at home and abroad is to improve postoperative shoulder motion and upper extremity function in patients with breast cancer randomized controlled study of quantitative analysis, so as to provide basis for the establishment of breast cancer patients postoperative exercise prescription.

INPLASY registration number: This protocol was registered with the International Platform of Registered Systematic Review and Meta-Analysis Protocols (INPLASY) on 06 June 2021 and was last updated on 06 June 2021 (registration number INPLASY202160018).

\section{INTRODUCTION}

Review question / Objective: To systematically evaluate the intervention effect of physical exercise on shoulder range of motion and upper limb function in patients with breast cancer after surgery.

Condition being studied: Breast cancer (BC) is the most common disease in 
women and the leading cause of cancer death in women. With the progress of medical treatment, the survival rate of breast cancer patients after 5 years has been as high as $90 \%$, but the complications caused by surgery and chemotherapy are more serious.Studies have shown that up to $67 \%$ of breast cancer patients experience reduced shoulder mobility and upper limb dysfunction following treatment. Long-term dysfunction can lead to chronic pain, decreased muscle strength, I ym phedema, cardiopulmonary dysfunction, and even permanent disability.However, sports can improve shoulder joint activity and upper limb function in patients with breast cancer, therefore, proposed using Meta analysis method, the exercise therapy at home and abroad is to improve postoperative shoulder motion and upper extremity function in patients with breast cancer randomized controlled study of quantitative analysis, so as to provide basis for the establishment of breast cancer patients postoperative exercise prescription.

\section{METHODS}

Participant or population: After Surgery of Breast Cancer Patients.

Intervention: Physical Exercise.

Comparator: Shoulder motion; upper limb function.

Study designs to be included: Randomized controlled trials.

Eligibility criteria: Postoperative patients with breast cancer, regardless of race or nationality, age $\geq 18$ years old, no mental disorders or cardiopulmonary dysfunction.

Information sources: Randomized controlled trials on The intervention of exercise therapy on shoulder range of motion and upper limb function in patients with breast cancer after surgery were searched from Web of Science, PubMed, The Cochrane Library, Embase, CNKI, Wan Fang Data, and Sinomed databases, and
The retrieval period was from each database to February 16, 2021.

Main outcome(s): A total of 14 pieces of literature and 18 studies involving 1241 patients were included.Physical exercise can improve shoulder flexion (SMD $=0.53,95 \% \mathrm{Cl}(0.31,0.76), \mathrm{P}<0.00001)$. There was no significant difference in DASH score (MD $=-12.53,95 \%$ C I $(-35.13,10.10), P<0.00001)$, CMS score (SMD $=0.90,95 \% \mathrm{Cl}(0.57,1.24) . \mathrm{P}<0.00001)$ was of significant significance.

Quality assessment / Risk of bias analysis: According to the Cochrane manual recommended risk bias tool from random sequence generation, allocation concealment, subjects, and the researchers ending index blinded, blind method, the data is incomplete and selective reports and other bias to evaluate seven aspects, for each index using the low risk of bias, uncertainty "bias", to identify "high risk of bias".

Strategy of data synthesis: The data processing software was Reviewer Manager 5.3. This meta-analysis strictly followed the PRISMA guidelines. $P$ value and 12 were used for heterogeneity test. If there was statistical heterogeneity between study results $(12 \geq 50 \%, P<0.10)$, the random effect model was used, and the fixed effect model was used otherwise.MD and SMD were selected for calculation, and the $95 \%$ confidence interval was calculated.Sensitivity analysis was performed using a single study by elimination method Stata 14.0 was used to test for publication bias.

Subgroup analysis: Age; Time to start intervention after surgery; Form of movement; Movement cycle.

Sensitivity analysis: Sensitivity analysis was performed using a single study by elimination method.

Country(ies) involved: China. 
Keywords: exercise therapy; breast cancer; shoulder motion; upper limb function; meta-analysis.

Contributions of each author:

Author 1 - Cong Liu.

Author 2 - Rao Chen.

Author 3 - Xinhu Zheng.

Author 4 - Xing Wang. 